\title{
Using photoactivatable fluorescent protein Dendra2 to track protein movement
}

\author{
Dmitriy M. Chudakov, Sergey Lukyanov, and Konstantin A. Lukyanov
}

Institute of Bioorganic Chemistry, Russian Academy of Sciences, Moscow, Russia

Photoactivatable fluorescent proteins are capable of dramatic changes in fluorescent properties in response to specific light irradiation. For example, they can be converted from cyan to green, or from green to red, or from nonfluorescent to a brightly fluorescent state. Several types of such proteins were developed recently, and some of them are already becoming popular tools to study protein mobility. Here we provide detailed recommendations on application of the monomeric green-to-red photoconvertible fluorescent protein Dendra2 for protein tracking in living cultured cells.

\section{Introduction}

Green fluorescent protein (GFP) and its homologs from jellyfishes, anthozoans, and copepods represent a unique protein family capable of self-catalyzed formation of a chromogenic group within the protein globule. GFP-like proteins become fluorescent or colored after protein translation, requiring no external enzymes or cofactors except molecular oxygen. Perhaps, 99\% of GFP-related works utilize fluorescent protein markers allowing in vivo visualization of diverse cellular processes (1). In particular, photobleaching of a fluorescent protein fused to a protein of interest is a widely used technique for studies of protein mobility by tracking the photobleached and nonphotobleached protein exchange rate (2).

A few years ago, several laboratories in the world (including ours) began to work on the development of light-controlled fluorescent proteins, whose spectral properties can be directly changed by a pulse of light. Of course, fluorescence that can be switched on by light represents the strongest practical interest. A number of so-called photoactivatable fluorescent proteins (PAFP) capable of a marked increase in fluorescence brightness in response to irradiation with light of specific wavelength and intensity were developed (3) (Table 1).
PAFPs allow one to photolabel and track the movement of living cells, organelles, and intracellular molecules. Probably the most productive use of PAFPs is tracking of the redistribution of fusion protein(s) of interest. Here, a protein of interest, tagged with a PAFP, can be precisely photolabeled and tracked in a spatially and temporally controlled manner. By timing the motion, one could determine rates and preferred directions of protein movement inside living cells. The approach can be widely applied to estimate protein movement rates and to compare these rates as cells respond to various stimuli. PAFPs can also be targeted to particular organelles, to measure and compare the rates of protein movement in various compartments. They also allow one to study protein exchange between compartments, as well as fission and fusion of organelles. Fusion of membrane-associated proteins or short peptides with PAFPs allows researchers to study their lateral diffusion in, and the viscosity of, membranes.

A number of novel techniques have been developed using PAFPs for precise cellular studies. These include: (i) monitoring of protein turnover by tracking of degradation of the irreversibly photoactivated protein (4); (ii) monitoring of dynamic protein interactions with photoquenching fluorescence resonance energy transfer (FRET) (5); (iii) fast

Table 1. Properties of Selected Photoactivatable Fluorescent Proteins

\begin{tabular}{|lcccc|}
\hline Protein & Photoconversion & Activating Light & Quenching Light & Oligomeric State \\
\hline PA-GFP & None to Green & UV or Violet & - & Monomer \\
PS-CFP & Cyan to Green & UV or Violet & - & Monomer \\
Kaede & Green to Red & UV or Violet & - & Tetramer \\
KikGR & Green to Red & UV or Violet & -10 \\
EosFP & Green to Red & UV or Violet & - & Tetramer \\
mEosFP & Green to Red & UV or Violet & Tetramer \\
Dendra & Green to Red & UV or Violet or Blue & Monomer \\
KFPs & None to Red & Green & Blue & Monomer \\
Dronpa & None to Green & UV or Violet & Blue & Tetramers \\
PA-GFP, photoactivatable green fluorescent protein; PS-CFP, photoswitchable cyan fluorescent protein; KFPs, kindling fluorescent proteins. & 13 \\
\hline
\end{tabular}




\section{Tech Insight}

protein tracking by repeated reversible photoactivation and averaging of tracking series (6); and (iv) high-resolution imaging techniques, based on reversible (7) or irreversible (8) photoactivation.

Several types of PAFPs can be distinguished (Table 1). The first type includes photoactivatable GFP (PA-GFP) (9), photoswitchable cyan fluorescent protein (PS-CFP) (10) and its enhanced version PS-CFP2 (Evrogen, Moscow, Russia). All these PAFPs are mutant variants of natural GFPs from Aequorea victoria and Aequorea coerulescens. In these proteins, the chromophore initially exists exclusively in a neutral state with an absorption maximum at about $400 \mathrm{~nm}$. These initial forms are fluorescent, and excitation by a weak $400 \mathrm{~nm}$ light can be used for the preliminary visualization of PA-GFP (green emission peaked at $515 \mathrm{~nm}$ ), PS-CFP, and PS-CFP2 (cyan emission peaked at $468 \mathrm{~nm}$ ). Irradiation with more intense UV or violet light (350-420 nm) leads to the irreversible chromophore transition from a neutral to an anionic state, which results from light-driven decarboxylation of an inner glutamate residue (Glu222) (11). This transition is associated with 100- to 400-fold increase in absorption-excitation peak at about $500 \mathrm{~nm}$, with green emission at about $515 \mathrm{~nm}$. Compared to PA-GFP, PS-CFP2 provides easier primary visualization and higher photoactivation contrast.

The second PAFP type includes Anthozoa-derived greento-red convertible proteins and their enhanced variants. In the dark, these proteins mature (fold and form chromophore) up to the green fluorescent state, while irradiation with UV-violet light results in their irreversible transition into a red fluorescent state. The first member of this group was named Kaede (12). Later, EosFP and mEosFP (13), KikGR (14), Dendra (15), and its enhanced variant Dendra2 (Evrogen) were developed. These PAFPs are characterized with high contrasting ratiometric photoswitching (i.e., expressed increase of the red fluorescent signal is accompanied by an expressed decrease of the green fluorescence) and allow reliable visualization of the initial (nonactivated) protein form and tracking of both forms' redistribution after the activation.

All the PAFPs mentioned above demonstrate irreversible photoconversion. In contrast, reversibly convertible PAFPs ensure repeated activation and quenching. For example, chromoprotein asFP595 (16) as well as its mutants called kindling fluorescent proteins (KFPs) $(17,18)$ can be transformed from nonfluorescent to the red fluorescent state by irradiation with relatively intense green (or, for some variants, blue) light. After that, they spontaneously relax back to the dark state within tens of seconds or minutes. Alternatively, after photoactivation, most KFP variants can be instantly quenched by blue light. The proposed mechanism for these photoconversions is a light-induced trans-cis isomerization of the chromophore, which possesses higher fluorescence quantum yield in a cis conformation (18-25).
The so-called Dronpa (26) represents another type of reversible PAFP. Initially, Dronpa fluoresces green upon blue light excitation. Upon intense irradiation by blue light, Dronpa is quenched to the nonfluorescent form. After that, Dronpa can be activated back to the green fluorescent state by a pulse of UV-violet light. Several similarities with KFPs' photobehavior indicate that the mechanism for Dronpa photoactivation is likely cis-trans isomerization as well $(3,27)$. Solutions of crystal structures $(27,28)$ and biophysical studies $(29,30)$ should help reveal the basis for this phenomenon.

As with other common fluorescent proteins, the oligomeric state of many PAFPs restricts the range of their applications. In most cases, attempts to use tetrameric GFP-like proteins to label cellular proteins result in disturbance of the studied protein's function, mislocalization, and aggregation effects. Only monomeric PAFPs: PA-GFP, PS-CFP, PS-CFP2, mEosFP, Dendra, Dendra2, and Dronpa are widely suitable for the labeling and tracking of the target proteins. Oligomeric PAFPs, such as Kaede, KikGR, EosFP, and KFP1 can be utilized mainly for photolabeling organelles and cells.

A

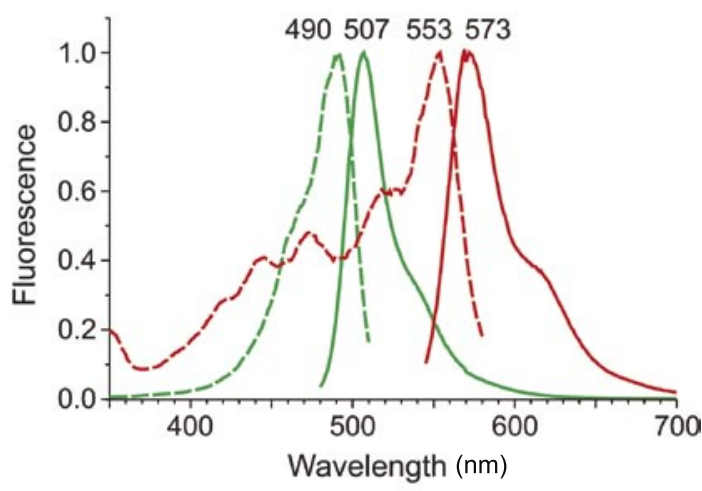

B

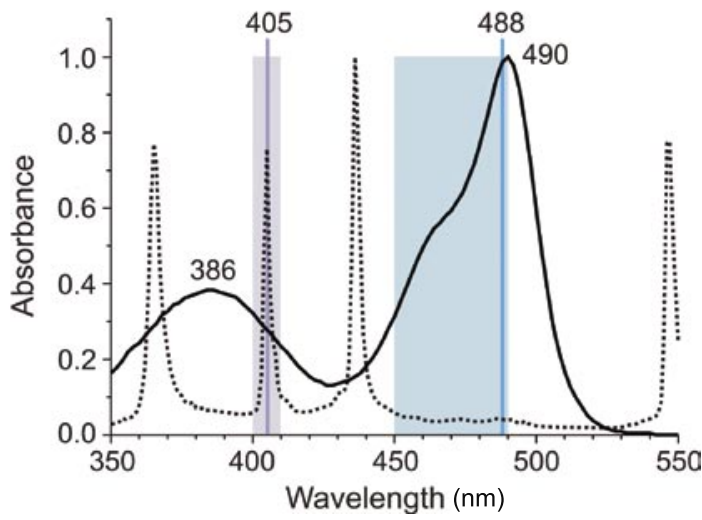

Figure 1. Spectral properties of Dendra2. (A) Normalized excitation (dashed lines) and emission (solid lines) spectra of green and red states of Dendra2. (B) Absorption spectrum of Dendra2 (solid line) is shown over the standard emission spectrum of mercury lamp (dotted line). The recommended excitation filters bandpass (violet and blue rectangles) and laser lines (violet and blue vertical lines) are shown. 


\section{Dendra2: a Monomeric Green-to-Red Photoconvertible Protein}

In the present paper, we are focusing on the detailed description of Dendra2-an improved commercially available version of green-to-red convertible protein Dendra. Compared to Dendra, Dendra2 comprises a single A224V substitution, which leads to more complete maturation (chromophore formation) and a brighter fluorescence both before and after photoswitching. In contrast to all other monomeric PAFPs, which necessarily require UV-violet (e.g., $405 \mathrm{~nm}$ laser) light for activation, Dendra and Dendra2 permit the use of blue (e.g., $488 \mathrm{~nm}$ laser) activating light.

The key desirable properties of any fluorescent protein are: bright fluorescence, monomeric nature (making it suitable for fusion protein labeling), fast maturation (important for early signal detection and for overall brightness in a cell, which is dependent on the balance between protein maturation and turnover rate), high photostability (allowing prolonged and/or high intensity excitation for the visualization of minor amounts of the protein and for time series), and high $\mathrm{pH}$ stability (making the fluorescent signal independent of local $\mathrm{pH}$ changes and allowing targeting of the protein to acidic organelles without signal loss).

Among the photoactivatable proteins, Dendra2 provides a unique combination of advantageous properties: monomeric state suitable for protein labeling, fast and efficient

A

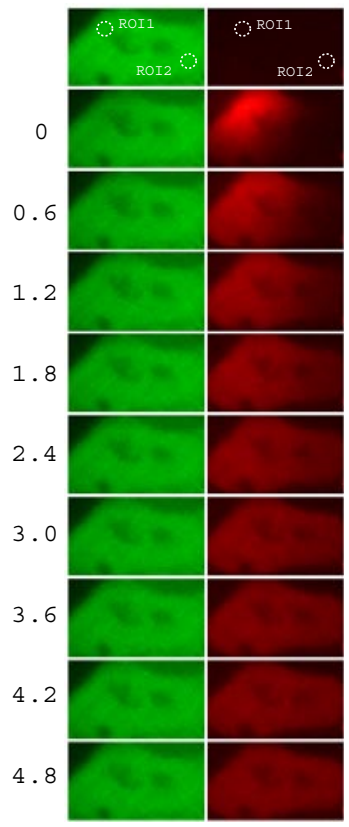

B

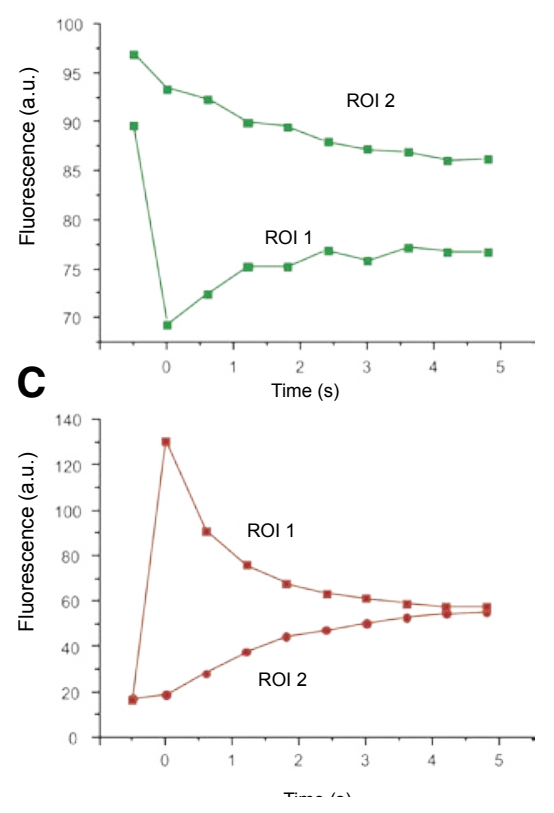

Figure 2. Tracking of Dendra2 fast redistribution within HeLa nucleus. (A) Time series of the photoactivated protein redistribution. Time, in seconds, is shown on the left. Left column, green channel; right column, red channel. Time zero is set at the commencement of photoactivation. (B) Redistribution of the green signal for region of interest (ROI) 1 (photoactivation point) and ROI 2. (C) Redistribution of the red signal. a.u., arbitrary units. maturation at $37^{\circ} \mathrm{C}$ in mammalian cells, high contrast photoconversion, bright fluorescence of both initial and photoactivated forms, high photostability of the activated red signal, and low phototoxic activation with the 488$\mathrm{nm}$ laser commonly installed on all commercial confocal microscopes. The minor disadvantage that should be kept in mind is a relatively low pH stability of the activated red form (with a pKa, i.e., the $\mathrm{pH}$ at which fluorescence is $50 \%$ of maximal, being 6.9). Here we provide some practical recommendations concerning applications of Dendra2 for tracking proteins of interest.

\section{Primary Visualization of Dendra2}

Nonactivated Dendra2 possesses excitation-emission maxima at 490 and $507 \mathrm{~nm}$ (Figure 1A). Therefore, the 488-nm laser line perfectly fits the excitation maximum ensuring easy detection of the green signal using confocal microscopes. Commonly used fluorescence filter sets for enhanced GFP (EGFP), fluorscein isocytothiocynate (FITC), and other green dyes are well suited to visualize Dendra2's green state. However, wide-field arc lamp excitation with a blue light can cause undesirable photoconversion of the whole visualized field and therefore should be very accurately applied for the preliminary visualization of Dendra2. Therefore, we recommend using strongly attenuated light from a mercury lamp and avoiding prolonged exposure of Dendra2-expressing cells.

Optimally, we recommend using confocal scanning with $488 \mathrm{~nm}$ excitation light for the visualization of nonphotoconverted Dendra2. Short pixel dwelling time (the time the laser spends at every point) in the course of commonly applied medium speed scanning $(400-800 \mathrm{~Hz})$ by a 488-nm laser line excitation results in zero or negligible photoactivation. In a confocal mode, a low-intensity (0.1\%-3\% power) $488-n m$ laser should be used to excite the green fluorescent form (detection at 500-550 nm). A control image in the red channel (excitation by 543$\mathrm{nm}$ laser, detection at 550-670 nm) shows a red signal, which should be close to zero level, before photoactivation. Ideally, you should obtain a clear cell image in the green channel and no signal above background in the red channel. If you see the same pattern for green and red fluorescence in the cell(s) of interest, this indicates that Dendra2 photoconversion has already occurred during the primary visualization. In this case, you should change the field of view using continuous scanning with 488-nm laser, to find Dendra2-expressing cells non-irradiated with a mercury lamp.

\section{Photoactivation of Dendra2}

The nonactivated Dendra2 absorption spectrum possesses peaks at 386 and $490 \mathrm{~nm}$ corresponding to neutral and anionic GFP-like chromophore states (Figure 1B). Excitation at the shorter wavelength peak (e.g., 405-nm laser) 
leads to a very efficient conversion of Dendra2 into the red fluorescent state, similarly to other Kaede-like proteins. We recommend applying the 405-nm line from a mercury lamp or diode laser to activate Dendra2 in the UV-violet region (see Examples 1 and 2 below). Shorter wavelength light (e.g., $366 \mathrm{~nm}$ ) can be used as well, but this irradiation is more harmful for cells.

Alternatively, Dendra2 can be activated by irradiation with blue light corresponding to its absorption peak at 490 $\mathrm{nm}$ (see Example 3). Compared to UV-violet, blue light activation has both advantages and disadvantages. Blue light is less harmful for living cells. Also, 488-nm lasers are more widely available in confocal microscopes. At the same time, Dendra2 activation with blue light is much less efficient than that with UV-violet light. Careful adjustment of several key parameters is necessary to obtain reliable photoconversion by a 488-nm laser.

This photoconversion appears to be a complex photochemical process, presumably requiring sequential absorption of two photons during some short time interval (15). Thus, the key features of the activating blue light are its intensity and continuity. Only continuous light of sufficient intensity can induce green to red conversion. At the same time, too strong irradiation may lead to bleaching of newly formed red fluorophores. In our experience, a mercury lamp (FITC filter set or similar) represents an efficient blue light source for Dendra2 photoconversion. In a confocal mode, two parameters of the 488-nm laser line are crucial: power and dwell time per pixel (the time that the laser spends at every point). An experimenter should vary these parameters and select optimal conditions for each experimental setup. The most efficient photoconversion occurs in a point bleach mode, when the laser beam stands still at a single pixel for some period of time (50-500 ms). For effective photoconversion in a scanning mode, we strongly recommend using a slow scan zoom mode to apply light onto a chosen cell region.

\section{Tracking Dendra2 After Activation}

Activated Dendra2 possesses excitation-emission maxima at 553 and $573 \mathrm{~nm}$, respectively. In a confocal mode, the red fluorescent signal can be acquired using 532-, 543-, 561-, or 568-nm excitation laser lines and detected at 550(570)-670 nm. For fast tracking (within the first several seconds after photoconversion), one should immediately start a time series of images to provide information on migration of the red Dendra2 from the region of activation. Since green fluorescence of Dendra2 decreases significantly upon its photoconversion to the red fluorescent form, one can follow migration of nonactivated green fluorescent Dendra2 into the region of activation (similarly to the photobleaching techniques). However, it is important, for such tracking, that a significant portion of the protein should be photoswitched from green to the red fluorescent form to allow monitoring of the green form redistribution.

Preferably, red and green signals from Dendra2 (as well as from any Kaede-like PAFP) should be collected separately, or in a sequential mode, for the clear separation of the two signals, because of the minor emission crosstalk of green fluorescence into the red channel. However, for fast dualcolor monitoring of both signals, simultaneous visualization mode can be used as well, using excitation with 488-nm and, for example, 561-nm laser lines.

The time interval between the images should be selected depending on the speed of the target protein movement. Based on the proposed rate of protein redistribution, or on the preliminary data concerning this rate, the frequency of image acquisition within a time series should be reduced as much possible. Indeed, for long time series, too often image acquisition will require many images to be captured, leading to undesirable photobleaching of the photoactivated protein. Generally, 10-30 consecutive images are enough to measure protein mobility and at the same time to avoid undesirable photobleaching/photoconversion effects during visualization.

\section{Examples of Dendra2 Tracking}

Here we provide examples of photoactivation and tracking of Dendra2 using different laser scanning confocal microscopes.

Example 1: tracking of Dendra2 fast redistribution within HeLa nucleus; photoactivation by $405-\mathrm{nm}$ laser. A Zeiss LSM 5 LIVE Duoscan confocal microscope with Plan Neofluar $40 \times / 1.3$ oil objective (Carl Zeiss, Jena, Germany) was used. HeLa cells were transiently transfected with pDendra2-C vector (Evrogen). Photoactivation was performed at the edge of the HeLa cell nucleus by a short pulse of 405-nm laser light. Further time series was obtained, 1 frame $/ 0.6 \mathrm{~s}$, in two channels: channel 1 , excitation $532 \mathrm{~nm}$ 8\%, emission collected BP 560-675; channel 2, excitation $488 \mathrm{~nm} 0.1 \%$, emission collected BP 495-555. Diffusion of the photoactivated red fluorescent protein within the nucleus could be accurately monitored, along with its replacement with the nonactivated green fluorescent form (Figure 2).

Example 2: tracking of Dendra2 redistribution from nucleus to cytosol of HeLa cell; photoactivation by $405-\mathrm{nm}$ laser. An Olympus FluoView ${ }^{\mathrm{TM}}$ FV1000 with objective UPLSAPO $60 \times$ O NA:1.35 (Olympus, Tokyo, Japan) was used. HeLa cells were transiently transfected with pDendra2-C vector. The protein was photoactivated in the center of nucleus by a 15\% 405-nm laser line, SIM Tornado (Olympus) for $200 \mathrm{~ms}$. Further redistribution of both protein forms was tracked. We used the following settings to obtain a time series ( 1 frame/20 s): zoom $2.4(88.064 \times 88.064 \mu \mathrm{m}$ image size); $1024 \times 1024$ pixels; sampling speed $2 \mu \mathrm{s}$ per pixel; line sequential mode: channel 1 , excitation with 


\section{Tech Insight}

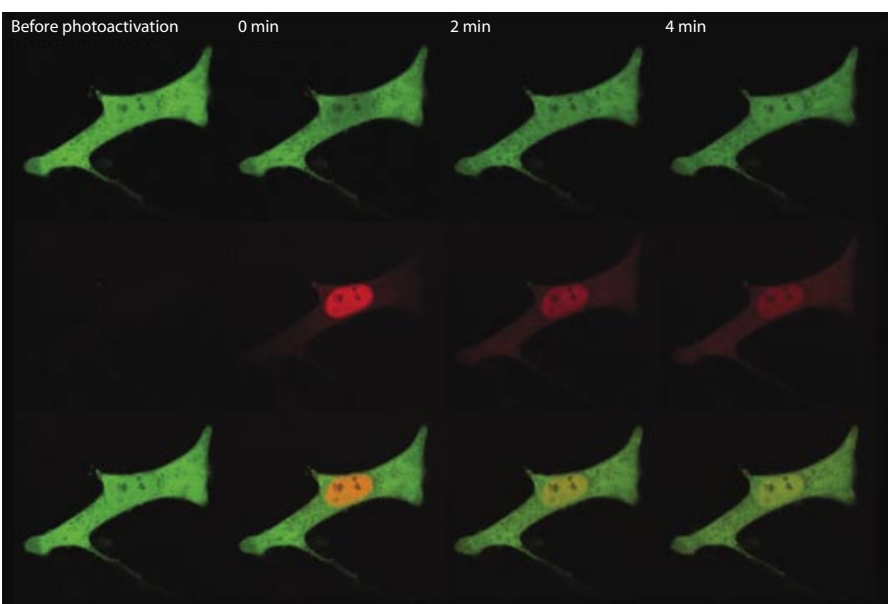

Figure 3. Tracking of Dendra2 redistribution from nucleus to cytosol of HeLa cell. Selected snapshots of the photoactivated protein redistribution. Time, in minutes, is shown on the top. First row, green channel; second row, red channel; third row, overlay. Time zero is set at the commencement of photoactivation.
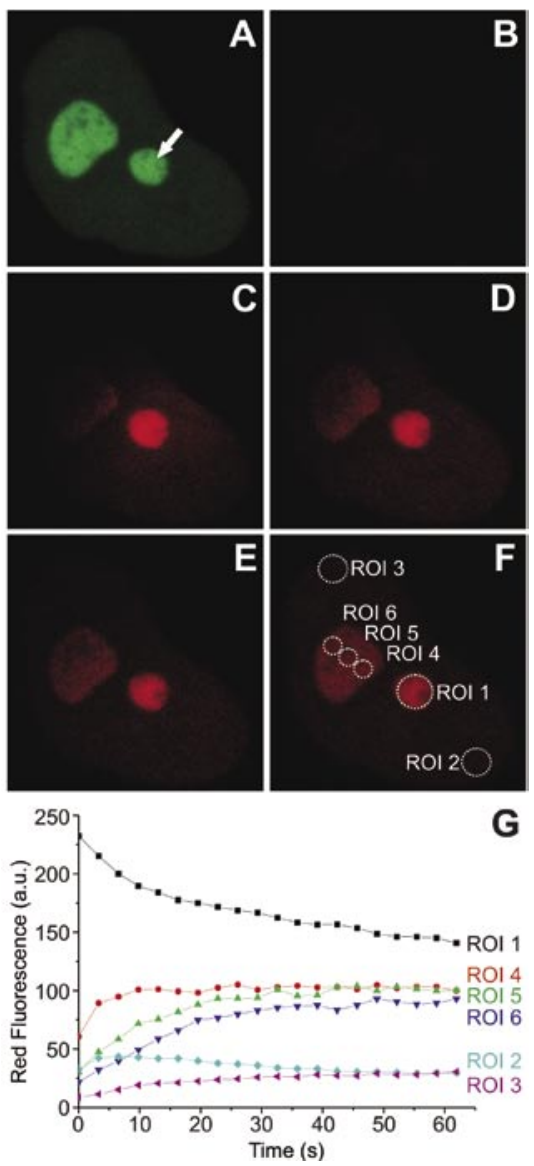

Figure 4. Tracking Dendra2-fibrillarin. (A-F) Confocal images of a single HeLa cell nucleus expressing Dendra2-fibrillarin fusion protein. ( $A$ and $B$ ) Green and red channels before photoactivation. (C-F) Red channel at 0, 6, 12 , and $60 \mathrm{~s}$ after photoactivation by $488-\mathrm{nm}$ laser at a point in one nucleolus (arrow). (G) Quantification of red signal in regions of interest (ROI) 1-6 designated on panel F. a.u., arbitrary units. 488-nm laser line, emission 500-541 nm; channel 2, excitation with 561-nm laser line, emission 576-676 nm; DM405/488/561/633; integration count 3. Redistribution of both the photoactivated red fluorescent protein and the nonactivated green fluorescent form between the nucleus and cytosol was observed (Figure 3).

\section{Example 3: activation and tracking Dendra2 fused with} nucleolus protein fibrillarin; photoactivation by $488-\mathrm{nm}$ laser. This experiment was performed using a Leica confocal inverted microscope DMIRE2 TCS SP2 equipped with HCX PL APO Ibd.BL $63 \times 1.4$ NA oil objective (Leica, Wetzlar, Germany) and $125 \mathrm{~mW}$ Ar and $1 \mathrm{~mW}$ HeNe lasers. Figure 4 shows Dendra2-fibrillarin tracking in nucleus of a HeLa cell transiently transfected with pDenrda2-fibrillarin vector (Evrogen). We used the following settings: mode, xyt; format, $512 \times 512$ pixels; zoom, $13(18 \times 18 \mu \mathrm{m}$ field of view); scan speed, $400 \mathrm{~Hz}$; beam expander, 3; pinhole, $140 \mu \mathrm{m}$; laser, $488 \mathrm{~nm} \mathrm{1 \%}$ power, PMT1, 500-535 nm, gain $725 \mathrm{~V}$ (for green fluorescence detection); or laser, $543 \mathrm{~nm} \mathrm{20 \%}$ power, PMT2, 560-680 nm; gain $700 \mathrm{~V}$ (for red fluorescence detection). Activation was done at a point within smaller nucleolus using point bleach mode by $25 \%$ 488-nm laser for $200 \mathrm{~ms}$. After that, 20 images in red channel were taken with 3-s time interval. As a result, we were able to observe a drastic increase of red signal in the activated nucleolus [region of interest (ROI) 1] and further migration of red signal in the nucleoplasm and adjacent nucleolus. A transient wave of Dendra2-fibrillarin migration was clearly detected in a nucleoplasm region near the activation point (ROI 2); only in about $45 \mathrm{~s}$ red signal became equalized across the nucleoplasm (compare $\mathrm{ROI} 2$ and 3). Analysis of red signal within the nonactivated nucleolus showed that Dendra2-fibrillarin easily migrates through the nucleolus with a rate comparable to that in the nucleoplasm. So, Dendra2-fibrillarin accumulation occurred first at the side closest to the activated nucleoIus (ROI 4), then in the central part (ROI 5), then at the opposite side (ROI 6), but not first at the periphery, then in the central part (as it could be expected). Apparently, such detailed information about migration of fibrillarin in nucleus cannot be obtained in a single experiment using classical approaches based on photobleaching.

\section{Acknowledgments}

We are grateful to T. Zimmermann (Centre de Regulació Genòmica, Barcelona, Spain), D. Ossipov and W. Hempell (Olympus), R. Wolleschensky and M. Kempe (Zeiss), and J. Schroeder (Leica-Microsystems). This work was supported by Russian Academy of Sciences for the program Molecular and Cell Biology, EC FP-6 Integrated Project LSHG-CT-2003-503259, National Institutes of Health (GM070358). D.M.C. and K.A.L. are supported by Grants of the President of Russian Federation MK-8236.2006.4 and Russian Science Support Foundation. 


\section{References}

1.Chudakov, D.M., S. Lukyanov, and K.A. Lukyanov. 2005. Fluorescent proteins as a toolkit for in vivo imaging. Trends Biotechnol. 23:605-613.

2.Lippincott-Schwartz, J., N. Altan-Bonnet, and G.H. Patterson. 2003. Photobleaching and photoactivation: following protein dynamics in living cells. Nat. Cell Biol. Suppl:S7-S14.

3.Lukyanov, K.A., D.M. Chudakov, S. Lukyanov, and V.V. Verkhusha. 2005. Innovation: photoactivatable fluorescent proteins. Nat. Rev. Mol. Cell Biol. 6:885-891.

4.Zhang, L., N.G. Gurskaya, E.M. Merzlyak, D.B. Stanoverov, N.N. Mudrik, O.N. Samarkina, L.M. Vinokurov, S. Lukyanov, and K.A. Lukyanov. 2007. Method for real-time monitoring of protein degradation at the single cell level. BioTechniques 42:446450 .

5.Demarco, I.A., A. Periasamy, C.F. Booker, and R.N. Day. 2006. Monitoring dynamic protein interactions with photoquenching FRET. Nat. Methods 3:519-524.

6.Chudakov, D.M., T.V. Chepurnykh, V.V. Belousov, S. Lukyanov, and K.A. Lukyanov. 2006. Fast and precise protein tracking using repeated reversible photoactivation. Traffic 7:13041310.

7.Hofmann, M., C. Eggeling, S. Jakobs, and S.W. Hell. 2005. Breaking the diffraction barrier in fluorescence microscopy at low light intensities by using reversibly photoswitchable proteins. Proc. Natl. Acad. Sci. USA 102:17565-17569.

8.Betzig, E., G.H. Patterson, R. Sougrat, O.W. Lindwasser, S. Olenych, J.S. Bonifacino, M.W. Davidson, J. Lippincott-Schwartz, and H.F. Hess. 2006. Imaging intracellular fluorescent proteins at nanometer resolution. Science 313:1642-1645.

9.Patterson, G.H. and J. Lippincott-Schwartz. 2002. A photoactivatable GFP for selective photolabeling of proteins and cells. Science 297:1873-1877.

10. Chudakov, D.M., V.V. Verkhusha, D.B. Staroverov, E.A. Souslova, S. Lukyanov, and K.A. Lukyanov. 2004. Photoswitchable cyan fluorescent protein for protein tracking. Nat. Biotechnol. 22:1435-1439.

11. van Thor, J.J., T. Gensch, K.J. Hellingwerf, and L.N. Johnson. 2002. Phototransformation of green fluorescent protein with UV and visible light leads to decarboxylation of glutamate 222. Nat. Struct. Biol. 9:37-41.

12. Ando, R., H. Hama, M. Yamamoto-Hino, H. Mizuno, and A. Miyawaki. 2002. An optical marker based on the UV-induced green-to-red photoconversion of a fluorescent protein. Proc. Natl. Acad. Sci. USA 99:12651-12656.

13. Wiedenmann, J., S. Ivanchenko, F. Oswald, F. Schmitt, C. Rocker, A. Salih, K.D. Spindler, and G.U. Nienhaus. 2004. EosFP, a fluorescent marker protein with UV-inducible greento-red fluorescence conversion. Proc. Natl. Acad. Sci. USA 101:15905-15910.

14. Tsutsui, H., S. Karasawa, H. Shimizu, N. Nukina, and A. Miyawaki. 2005. Semi-rational engineering of a coral fluorescent protein into an efficient highlighter. EMBO Rep. 6:233-238.

15. Gurskaya, N.G., V.V. Verkhusha, A.S. Shcheglov, D.B. Staroverov, T.V. Chepurnykh, A.F. Fradkov, S. Lukyanov, and K.A. Lukyanov. 2006. Engineering of a monomeric greento-red photoactivatable fluorescent protein induced by blue light. Nat. Biotechnol. 24:461-465.

16. Lukyanov, K.A., A.F. Fradkov, N.G. Gurskaya, M.V. Matz, Y.A. Labas, A.P. Savitsky, M.L. Markelov, A.G. Zaraisky, et al. 2000. Natural animal coloration can be determined by a nonfluorescent green fluorescent protein homolog. J. Biol. Chem. 275:25879-25882.

17. Chudakov, D.M., V.V. Belousov, A.G. Zaraisky, V.V. Novoselov, D.B. Staroverov, D.B. Zorov, S. Lukyanov, and K.A. Lukyanov.
2003. Kindling fluorescent proteins for precise in vivo photolabeling. Nat. Biotechnol. 21:191-194.

18.Chudakov, D.M., A.V. Feofanov, N.N. Mudrik, S. Lukyanov, and K.A. Lukyanov. 2003. Chromophore environment provides clue to "kindling fluorescent protein" riddle. J. Biol. Chem. 278:7215-7219.

19. Wilmann, P.G., J. Petersen, R.J. Devenish, M. Prescott, and J. Rossjohn. 2005. Variations on the GFP chromophore: a polypeptide fragmentation within the chromophore revealed in the 2.1-A crystal structure of a nonfluorescent chromoprotein from Anemonia sulcata. J. Biol. Chem. 280:2401-2404.

20.Quillin, M.L., D.M. Anstrom, X. Shu, S. O'Leary, K. Kallio, D.M. Chudakov, and S.J. Remington. 2005. Kindling fluorescent protein from Anemonia sulcata: dark-state structure at 1.38 A resolution. Biochemistry 44:5774-5787.

21. Yampolsky, I.V., S.J. Remington, V.I. Martynov, V.K. Potapov, S. Lukyanov, and K.A. Lukyanov. 2005. Synthesis and properties of the chromophore of the asFP595 chromoprotein from Anemonia sulcata. Biochemistry 44:5788-5793.

22. Schafer, L.V., G. Groenhof, A.R. Klingen, G.M. UlImann, M. Boggio-Pasqua, M.A. Robb, and H. Grubmuller. 2007. Photoswitching of the fluorescent protein asFP595: mechanism, proton pathways, and absorption spectra. Angew. Chem. Int. Ed. Engl. 46:530-536

23. Grigorenko, B., A. Savitsky, I. Topol, S. Burt, and A. Nemukhin. 2006. Ground-state structures and vertical excitations for the kindling fluorescent protein asFP595. J. Phys. Chem. B Condens. Matter Mater. Surf. Interfaces Biophys. 110:1863518640.

24. Amat, P., G. Granucci, F. Buda, M. Persico, and V. Tozzini. 2006. The chromophore of asFP595: a theoretical study. J. Phys. Chem. B Condens. Matter Mater. Surf. Interfaces Biophys. 110:9348-9353.

25.Andresen, M., M.C. Wahl, A.C. Stiel, F. Grater, L.V. Schafer, S. Trowitzsch, G. Weber, C. Eggeling, H. Grubmuller, S.W. Hell, and S. Jakobs. 2005. Structure and mechanism of the reversible photoswitch of a fluorescent protein. Proc. Natl. Acad. Sci. USA 102:13070-13074.

26. Ando, R., H. Mizuno, and A. Miyawaki. 2004. Regulated fast nucleocytoplasmic shuttling observed by reversible protein highlighting. Science 306:1370-1373.

27. Stiel, A.C., S. Trowitzsch, G. Weber, M. Andresen, C. Eggeling, S.W. Hell, S. Jakobs, and M.C. Wahl. 2006. 1.8 A bright-state structure of the reversibly switchable fluorescent protein Dronpa guides the generation of fast switching variants. Biochem. J. 402:35-42.

28. Wilmann, P.G., K. Turcic, J.M. Battad, M.C. Wilce, R.J. Devenish, M. Prescott, and J. Rossjohn. 2006. The 1.7 a crystal structure of Dronpa: a photoswitchable green fluorescent protein. J. Mol. Biol. 364:213-224.

29. Habuchi, S., P. Dedecker, J. Hotta, C. Flors, R. Ando, H. Mizuno, A. Miyawaki, and J. Hofkens. 2006. Photo-induced protonation/deprotonation in the GFP-like fluorescent protein Dronpa: mechanism responsible for the reversible photoswitching. Photochem. Photobiol. Sci. 5:567-576.

30.Dedecker, P., J. Hotta, R. Ando, A. Miyawaki, Y. Engelborghs, and J. Hofkens. 2006. Fast and reversible photoswitching of the fluorescent protein Dronpa as evidenced by fluorescence correlation spectroscopy. Biophys. J. 91:L45-L47. 